\title{
Constitution et exploitation d'une base de données d'états de mer le long des côtes françaises par simulation numérique sur 23 ans
}

\author{
Michel Benoit ${ }^{1}$, Florence Lafon ${ }^{1}$, Gérard Goasguen ${ }^{2}$ \\ ${ }^{1}$ EDF R\&D - LNHE, Ingénieurs-chercheurs \\ ${ }^{2}$ CETMEF - DELCE, Ingénieur
}

\section{Résumé}

Une base de données numériques d'états de mer a été constituée à partir de simulations effectuées avec le logiciel TOMAWAC sur la période 1979-2002. Les champs de vent utilisés en entrée sont issus de la réanalyse ERA-40 du Centre Européen de Prévision Météorologique à Moyen Terme (ECMWF). Des premiers traitements statistiques ont été effectués, permettant d'établir des cartes représentatives de la climatologie moyenne et des conditions de houle de tempête sur le plateau continental européen. Enfin, on propose deux méthodes d'estimation des valeurs extrêmes de hauteurs significatives de houle associées à des périodes de retour supérieures à la durée simulée. Ces deux méthodes d'extrapolation statistique sont comparées sur une sélection de points.

\section{$\underline{\text { Abstract }}$}

A numerical wave data-base has been built by hindcasting sea-state conditions over a period of 23 years, from 1979 to 2002, with the TOMAWAC wave model. The wind fields used as input data come from the reanalysis ERA-40 of the European Center for Medium-Range Weather Forecasts (ECMWF). Some statistical processing of the results have been performed, so as to build synthetic charts of the medium and severe wave conditions over the European continental shelf. Finally two different methods are proposed to estimate extreme wave heights with return periods larger than the simulated period. Both methods for statistical extrapolation are applied to a selection of points and compared.

\section{Mots clés}

Etats de mer, hindcast, atlas de houle, TOMAWAC, valeurs extrêmes.

\section{Introduction}

Une bonne connaissance de la climatologie des états de mer est essentielle pour la gestion des opérations en mer, pour le dimensionnement des aménagements offshore ou côtiers, pour l'étude et la définition des stratégies d'aménagement du littoral, ou encore pour l'évaluation du potentiel d'un site en énergie des vagues. En complément des campagnes de mesures de houle in situ, la simulation 
numérique apparaît comme une approche efficace pour constituer des séries de données de longues durées sur l'ensemble du littoral. Ainsi, EDF-LNHE et le CETMEF collaborent depuis plusieurs années pour constituer une base de données d'états de mer le long des côtes françaises, à partir de simulations de hindcast sur une période de 23 ans, de 1979 à 2002. On présente d'abord brièvement les phases de construction $(\S 2)$ et de validation des modèles numériques $(\S 3)$, puis les premiers résultats des traitements statistiques effectués, sous forme de cartes synthétiques représentatives de conditions de houle moyennes et sévères $(\S 4)$. Enfin, deux méthodes d'extrapolation statistique sont comparées sur une sélection de points $(\S 5)$.

\section{Construction de la base de données d'états de mer}

Les simulations sont effectuées avec le logiciel de modélisation des états de mer TOMAWAC ${ }^{1}$, développé par EDF-LNHE avec le soutien du CETMEF. TOMAWAC est un modèle dit de « troisième génération ", qui résout l'équation d'évolution de la densité spectro-angulaire d'action d'onde ${ }^{3,6}$.

On utilise pour cette application deux maillages emboîtés de type "éléments finis », avec des résolutions spatiales différentes. Le premier modèle (dit « océanique ») couvre la partie nord de l'Océan Atlantique, avec une résolution spatiale de 1 degré au large et $20 \mathrm{~km}$ le long des côtes françaises (cf. figure 1). Ce modèle est utilisé sans conditions de houle incidente aux limites, i.e. on suppose que tous les états de mer sont générés sur l'emprise couverte par le modèle et que toutes les frontières absorbent la houle sans réflexion.

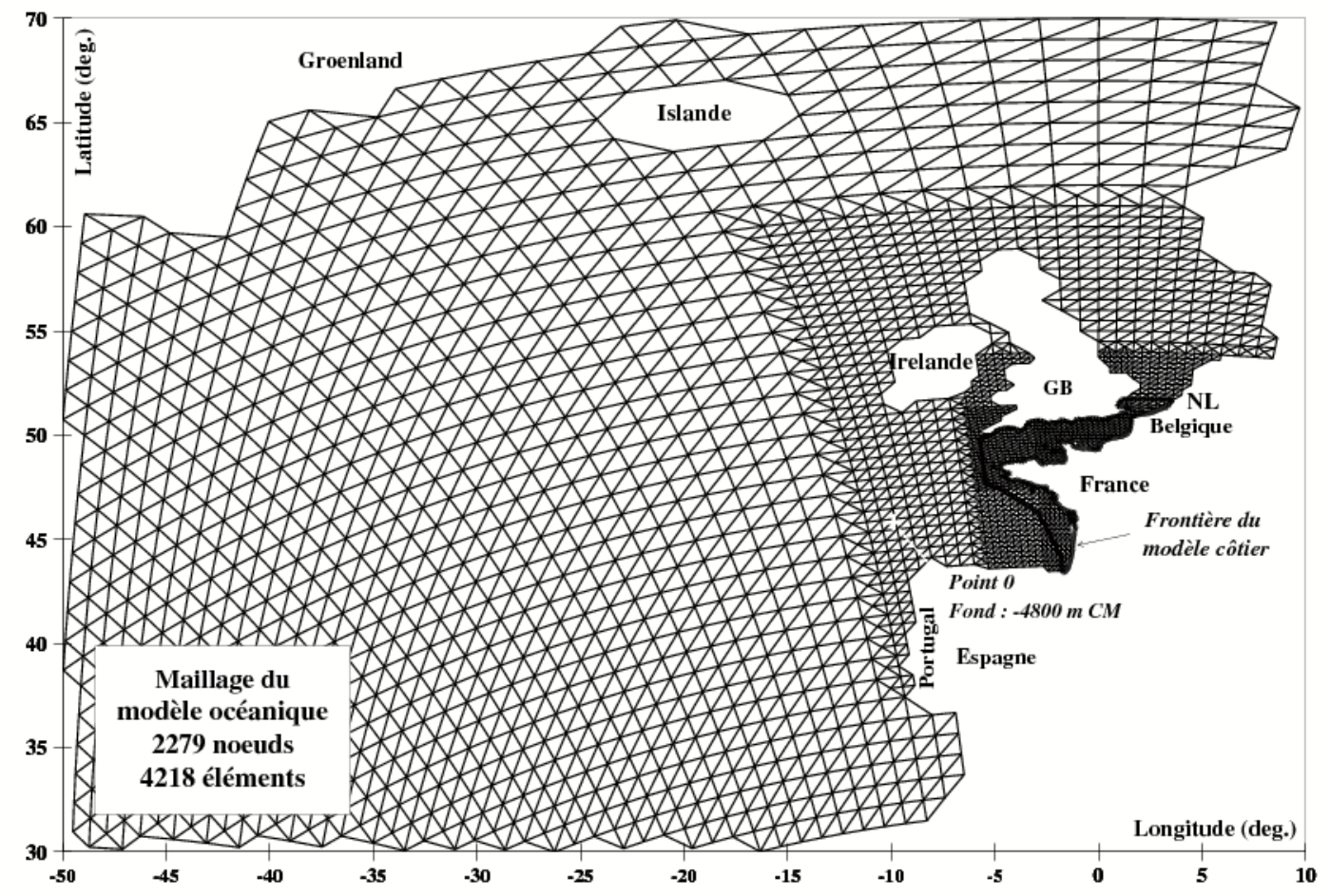

Figure 1 : Maillage du modèle océanique. 
Le second modèle (dit « côtier ») s'étend vers le large jusqu'à la limite du plateau continental, avec une résolution de quelques kilomètres près des côtes françaises (cf. figure 2). Ce modèle est forcé aux limites par les spectres directionnels de vagues calculés par le modèle océanique.

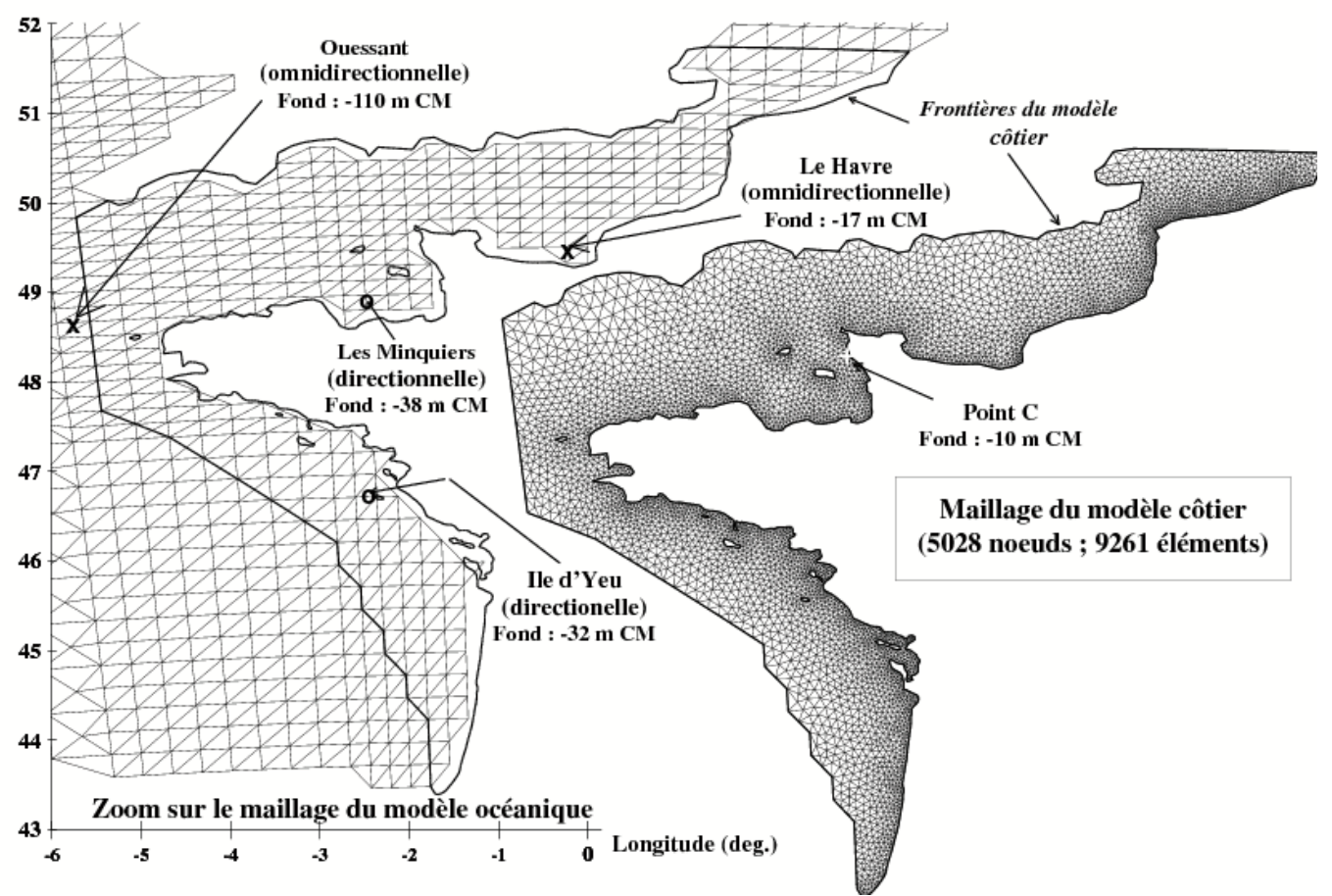

Figure 2: Maillage du modèle côtier (à droite), comparé au maillage du modèle océanique (à gauche) le long des côtes françaises.

Dans cette première version de la base de données d'états de mer, les simulations sont effectuées avec un niveau marin constant, correspondant au niveau moyen de marée. L'effet des courants n'est pas représenté. En revanche, les processus de réfraction, shoaling et frottement sur le fond sont activés dans les deux modèles de manière à tenir compte des effets de profondeur finie à l'approche des côtes. Le déferlement bathymétrique est pris en compte dans le modèle côtier.

On utilise comme forçage atmosphérique les champs de vent issus de la réanalyse ERA-40 du Centre Européen de Prévision Météorologique à Moyen Terme (ECMWF). Les champs de vent moyen à $10 \mathrm{~m}$ sont disponibles toutes les $6 \mathrm{~h}$ sur une grille rectangulaire de résolution 0.5 degré en latitude et en longitude.

\section{Validation par comparaison à des mesures de bouées sur 1999 et 2000}

Les deux modèles ont été calibrés et validés par comparaison avec les mesures de houlographes de la base de données CANDHIS du CETMEF, sur les années 1999 et $2000^{2}$. A titre d'exemple, on présente sur la figure 3 les diagrammes établissant la correspondance entre les quantiles (de $1 \%$ à $99 \%$ ) des séries temporelles de $\mathrm{H}_{\text {mo }}$ mesurée et calculée, pour les sites Ouessant, Ile d'Yeu, Les Minquiers et 
Le Havre (voir localisation figure 2). Les bouées «Ouessant» et « Ile d'Yeu» sont directement exposées au climat de houle de l'Océan Atlantique et situées dans des eaux profondes (Ouessant : $-110 \mathrm{~m} \mathrm{CM}$ ), à intermédiaires (Ile d'Yeu : $32 \mathrm{~m} \mathrm{CM}$ ). Les bouées «Les Minquiers » et «Le Havre » sont situées à l'intérieur de la Manche dans des eaux intermédiaires (Les Minquiers : $-38 \mathrm{~m} \mathrm{CM}$ ) à peu profondes (Le Havre : $-17 \mathrm{~m} \mathrm{CM}$ ).
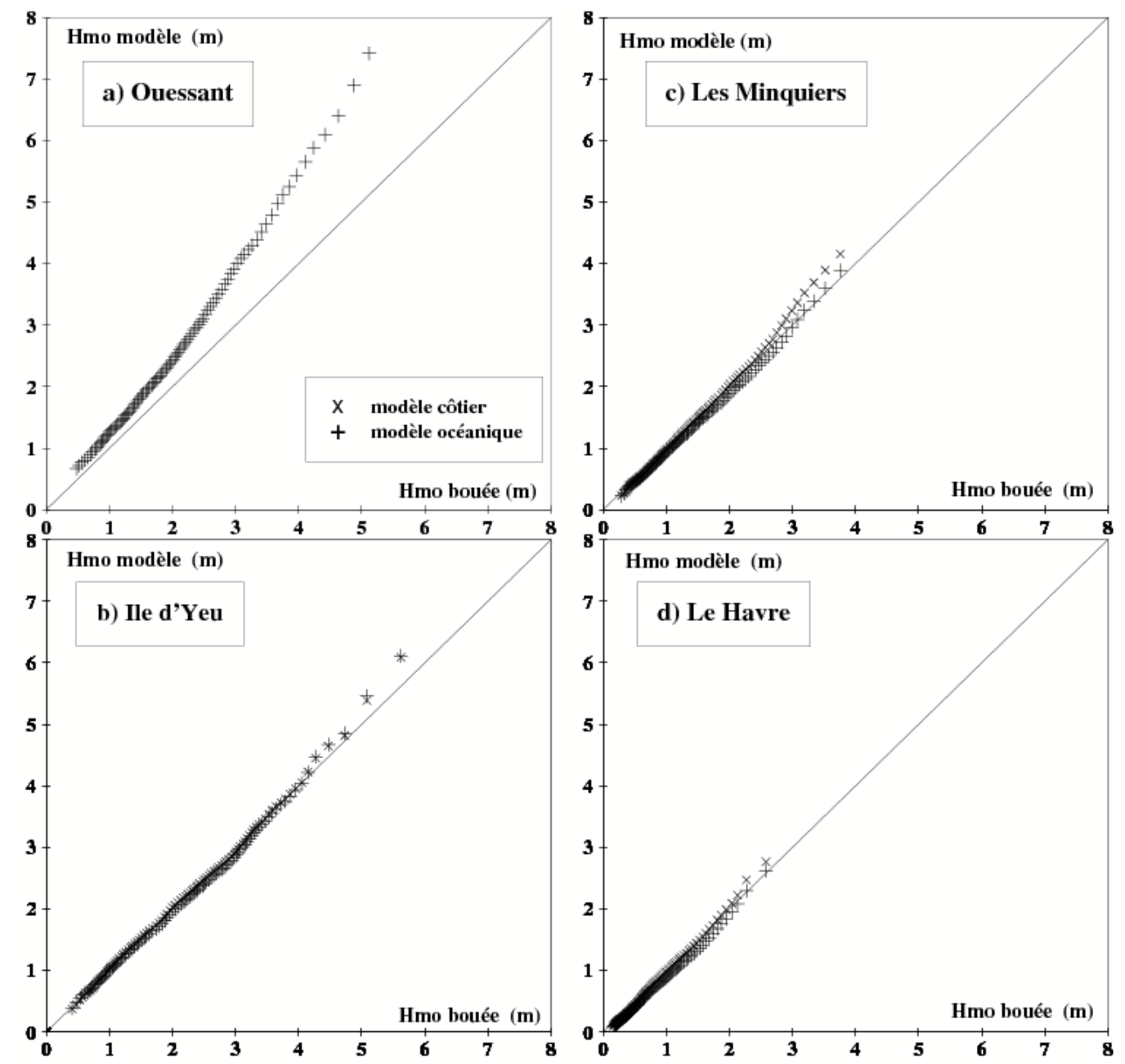

Figure 3 : Diagrammes quantile-quantile de $\mathrm{H}_{\mathrm{mo}}$ sur la période 1999-2000.

De manière générale, on observe une bonne adéquation entre les résultats des modèles et les mesures. A Ouessant, le biais positif apparent des modèles (cf. figure 3a) s'explique en partie par une tendance du capteur MRU monté sur la bouée-phare BEAIII à sous-estimer systématiquement les fortes valeurs de hauteurs de houle (tendance observée par le CETMEF lors de comparaisons avec une bouée Datawell en 2004). Le modèle côtier fournit des résultats très comparables à ceux du modèle océanique sur ces quatre sites, ce qui est logique pour les bouées «Ile d'Yeu » et «Les Minquiers », situées assez loin des côtes, mais plus remarquable pour «Le Havre ", où le modèle océanique fournit déjà de bons résultats malgré la profondeur relativement faible (-17 $\mathrm{m} \mathrm{CM})$. 


\section{Climat moyen d'états de mer le long des côtes françaises}

Les simulations avec les modèles océanique et côtier ont été effectuées à partir des champs de vent ERA-40 sur la période allant de Janvier 1979 à Août 2002, ce qui constitue une première version de la base numérique d'états de mer, d'une durée de 23.7 ans. Afin de ne pas introduire de biais saisonnier, on considère dans les analyses statistiques ci-après 23 années pleines, du 01/09/1979 au 31/08/2002.

Les paramètres réduits du spectre d'état de mer $\left(\mathrm{H}_{\mathrm{mo}}, \mathrm{T}_{\mathrm{m}}, \theta_{\mathrm{m}}\right.$, etc. $)$ sont stockés à chaque point des grilles de calcul avec un pas de temps horaire. On procède ensuite en chaque nœud à un traitement statistique automatisé des séries temporelles des paramètres réduits, de manière à déterminer les histogrammes, les distributions jointes $\left(\mathrm{H}_{\mathrm{mo}}, \mathrm{T}_{\mathrm{m}}\right),\left(\mathrm{H}_{\mathrm{mo}}, \theta_{\mathrm{m}}\right)$, etc. La figure 4 présente les cartes de la valeur moyenne (a) et du quantile à $99 \%$ (b) de la hauteur significative spectrale $\mathrm{H}_{\text {mo }}$, établies à partir des résultats du modèle océanique.

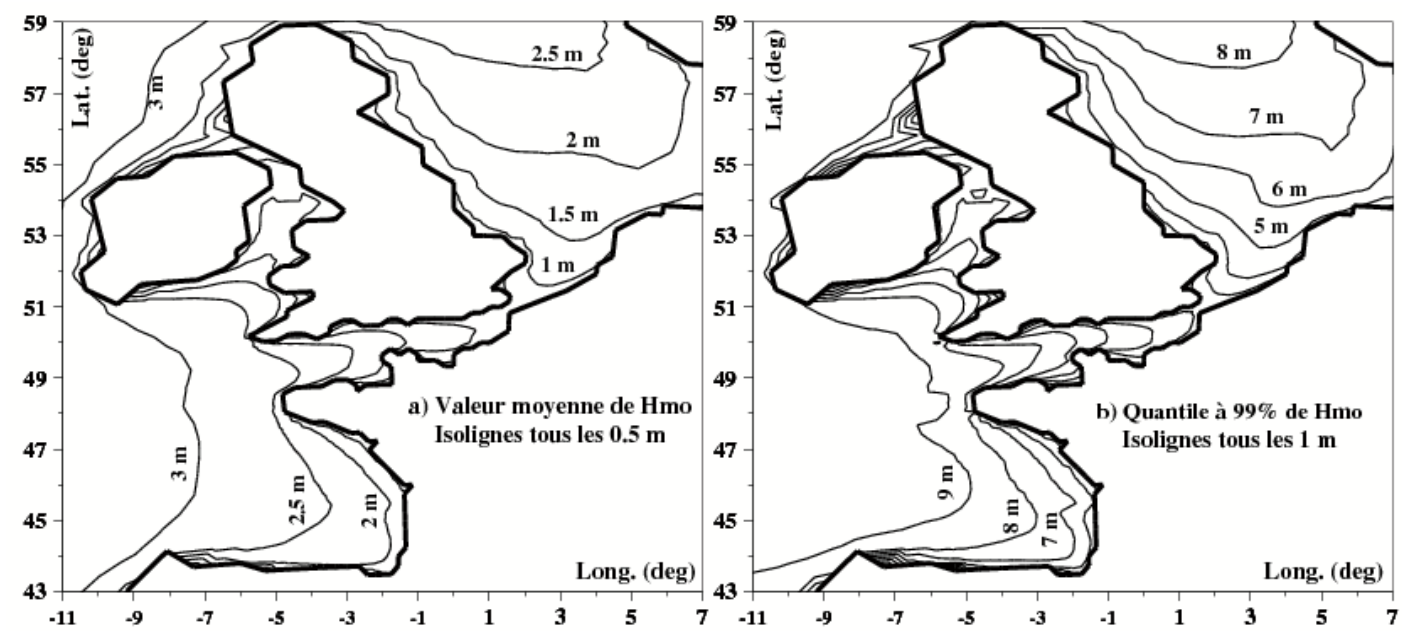

Figure 4 : Cartographie de la valeur moyenne (a) et du quantile à $99 \%$ (b) de $\mathrm{H}_{\text {mo }}$ obtenue avec le modèle océanique forcé par les vents ERA-40 sur la période 1979-2002.

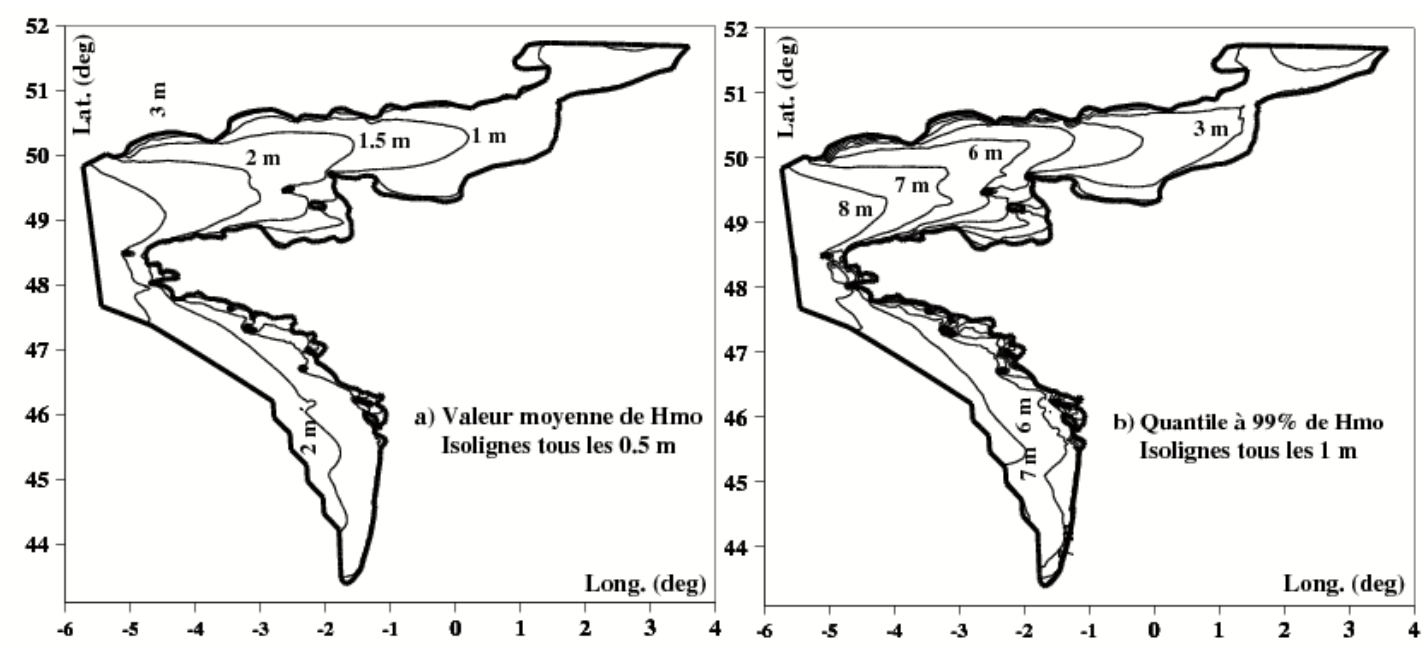

Figure 5 : Cartographie de la valeur moyenne (a) et du quantile à $99 \%$ (b) de $\mathrm{H}_{\text {mo }}$ obtenue avec le modèle côtier forcé par les vents ERA-40 sur la période 1979-2002. 
Les cartes de la figure 5 représentent les mêmes variables établies à partir des résultats du modèle côtier, avec une résolution plus fine autour des côtes françaises. Le quantile $99 \%$ correspond aux hauteurs significatives dépassées $1 \%$ $\mathrm{du}$ temps en moyenne : les cartes des figures $4 \mathrm{~b}$ et $5 \mathrm{~b}$ offrent donc une vue synthétique des conditions d'états de mer les plus sévères simulées sur la période 1979-2002 sur le plateau continental européen.

\section{Estimation des hauteurs extrêmes de houle par extrapolation statistique}

On cherche ici à estimer les hauteurs de vagues de périodes de retour supérieures à la durée simulée (23 ans). On considère pour cela deux méthodes d'extrapolation statistique, relevant de la théorie des valeurs extrêmes dans un cadre stationnaire asymptotique . $^{5}$

- La première méthode, dite « des maxima annuels » consiste à sélectionner la hauteur significative maximale enregistrée chaque année, puis à ajuster l'échantillon des maxima annuels à une loi généralisée de valeurs extrêmes (GEV), de la forme :

$$
\operatorname{GEV}(\mathrm{y})=\left\{\begin{array}{l}
\exp \left[-\left(1+\xi \frac{y-\mu}{\sigma}\right)^{-\frac{1}{\xi}}\right] \\
\exp \left[-\exp \left(-\frac{\mathrm{y}-\mu}{\sigma}\right)\right] \\
\text { si } \xi=0
\end{array} \text { où }\left\{\mathrm{y} \in \mathfrak{R}, 1+\xi\left(\frac{y-\mu}{\sigma}\right)>0\right\} \text { et } \mu \in \mathfrak{R}, \sigma>0, \xi \in \Re\right.
$$

- La deuxième méthode, dite «Peaks Over Threshold» (POT), consiste à sélectionner un échantillon de pics indépendants en utilisant une méthode à seuil. On suppose alors que les valeurs des pics sont réparties selon une distribution généralisée de Pareto (GPD), et que le processus d'occurence des pics est poissonien. La loi GPD s'écrit pour un seuil u donné :

$$
\operatorname{GPD}_{\mathrm{u}}(\mathrm{y})=\left\{\begin{array}{l}
1-\left(1+\xi \frac{y-u}{\sigma}\right)^{-\frac{1}{\xi}} \text { si } \xi \neq 0 \\
1-\exp \left(-\frac{y-u}{\sigma}\right) \text { si } \xi=0
\end{array} \text { où }\left\{\mathrm{y}>\mathrm{u}, 1+\xi\left(\frac{y-u}{\sigma}\right)>0\right\} \text { et } \sigma>0, \xi \in \mathfrak{R}\right.
$$

Les paramètres des lois GEV et GPD (paramètres de position $\mu$, d'échelle $\sigma$ et de forme $\xi$ ) sont estimés par la méthode du maximum de vraisemblance et un intervalle de confiance est calculé en appliquant la delta-méthode ${ }^{5}$.

Ces deux méthodes sont appliquées à 4 points : le point $\mathrm{O}$, situé au large du plateau continental (cf. figure 1), en grande profondeur $(-4800 \mathrm{~m} \mathrm{CM})$, les bouées « Ile d'Yeu » et « le Havre » et le point $\mathrm{C}$, situé près de la côte ouest du Cotentin (cf. figure 2) en faible profondeur $(-10 \mathrm{~m} \mathrm{CM})$. Le tableau 1 précise pour chaque point étudié le nombre de pics de l'échantillon de tempêtes sur la durée simulée de 23 années complètes, la valeur du seuil en approche POT et l'estimation du paramètre de forme $\xi$. 


\begin{tabular}{|c|c|c|c|c|}
\hline Site & Point O & Ile d'Yeu & Le Havre & Point C \\
\hline Cote du fond & $-4800 \mathrm{~m} \mathrm{CM}$ & $-32 \mathrm{~m} \mathrm{CM}$ & $-17 \mathrm{~m} \mathrm{CM}$ & $-10 \mathrm{~m} \mathrm{CM}$ \\
\hline \multicolumn{5}{|c|}{ Méthode des maxima annuels } \\
\hline Nombre de pics & 23 & 23 & 23 & 23 \\
\hline Paramètre de forme $\xi$ & 0.00 & -0.31 & -0.16 & -0.69 \\
\hline \multicolumn{7}{|c|}{ Méthode POT } \\
\hline Seuil & $8 \mathrm{~m}$ & $4 \mathrm{~m}$ & $2.5 \mathrm{~m}$ & $4 \mathrm{~m}$ \\
\hline Nombre de pics & 178 & 226 & 199 & 173 \\
\hline Paramètre de forme $\xi$ & 0.00 & -0.16 & -0.19 & -0.69 \\
\hline
\end{tabular}

Tableau 1 : caractéristiques principales des ajustements statistiques

On peut effectuer un diagnostic des ajustements à partir des diagrammes établissant la correspondance entre les quantiles de la loi théorique ajustée (GEV ou GPD selon le cas) et les quantiles de l'échantillon de tempêtes sélectionnées. Ces diagrammes sont représentés sur la figure 6 pour la méthode des maxima annuels et sur la figure 7 pour la méthode POT.
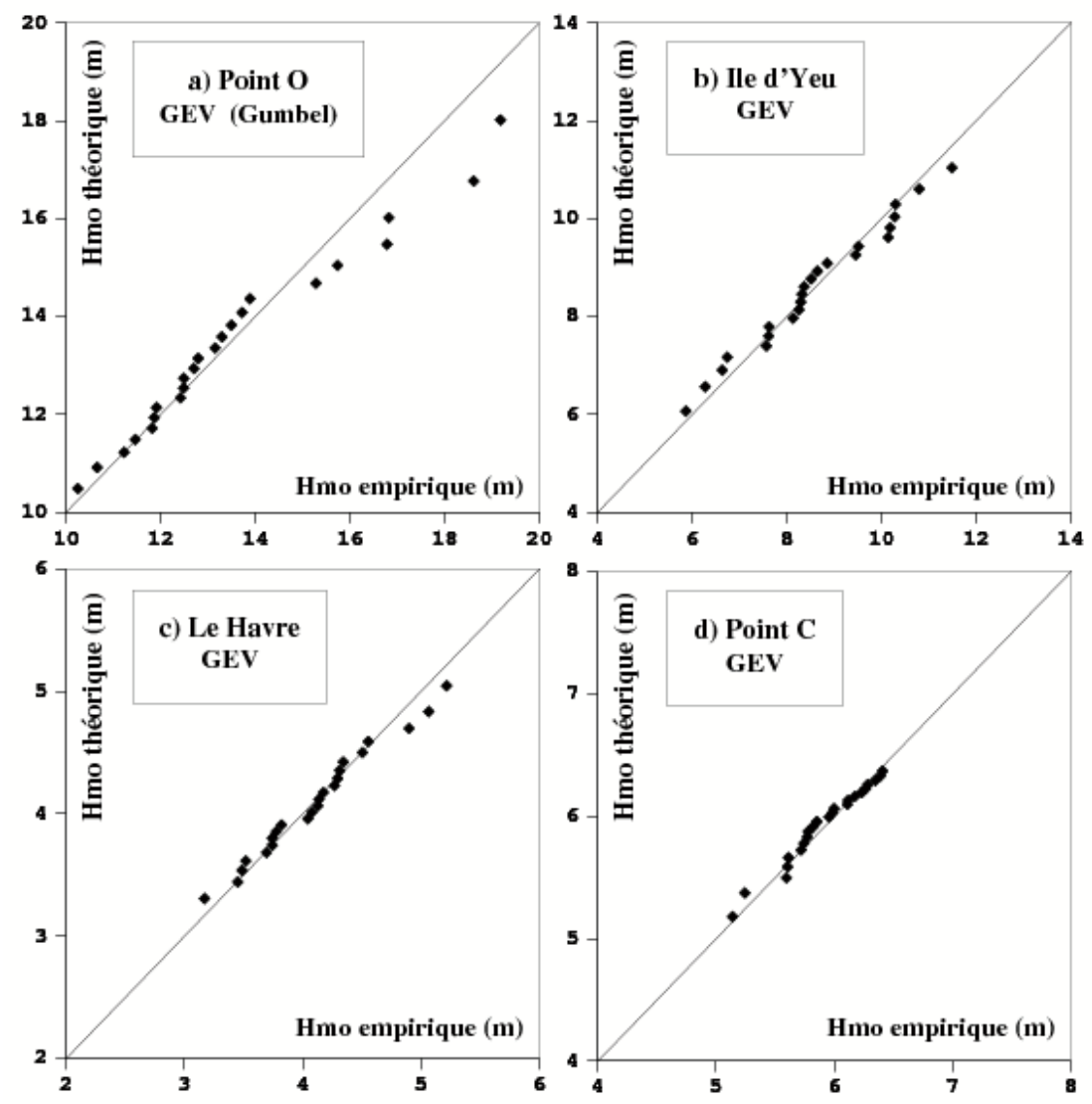

Figure 6 : Méthode des maxima annuels. Diagnostic de l'ajustement à une loi GEV en 4 sites : a) point océanique ; b) Ile d'Yeu ; c) Le Havre ; d) point côtier. 

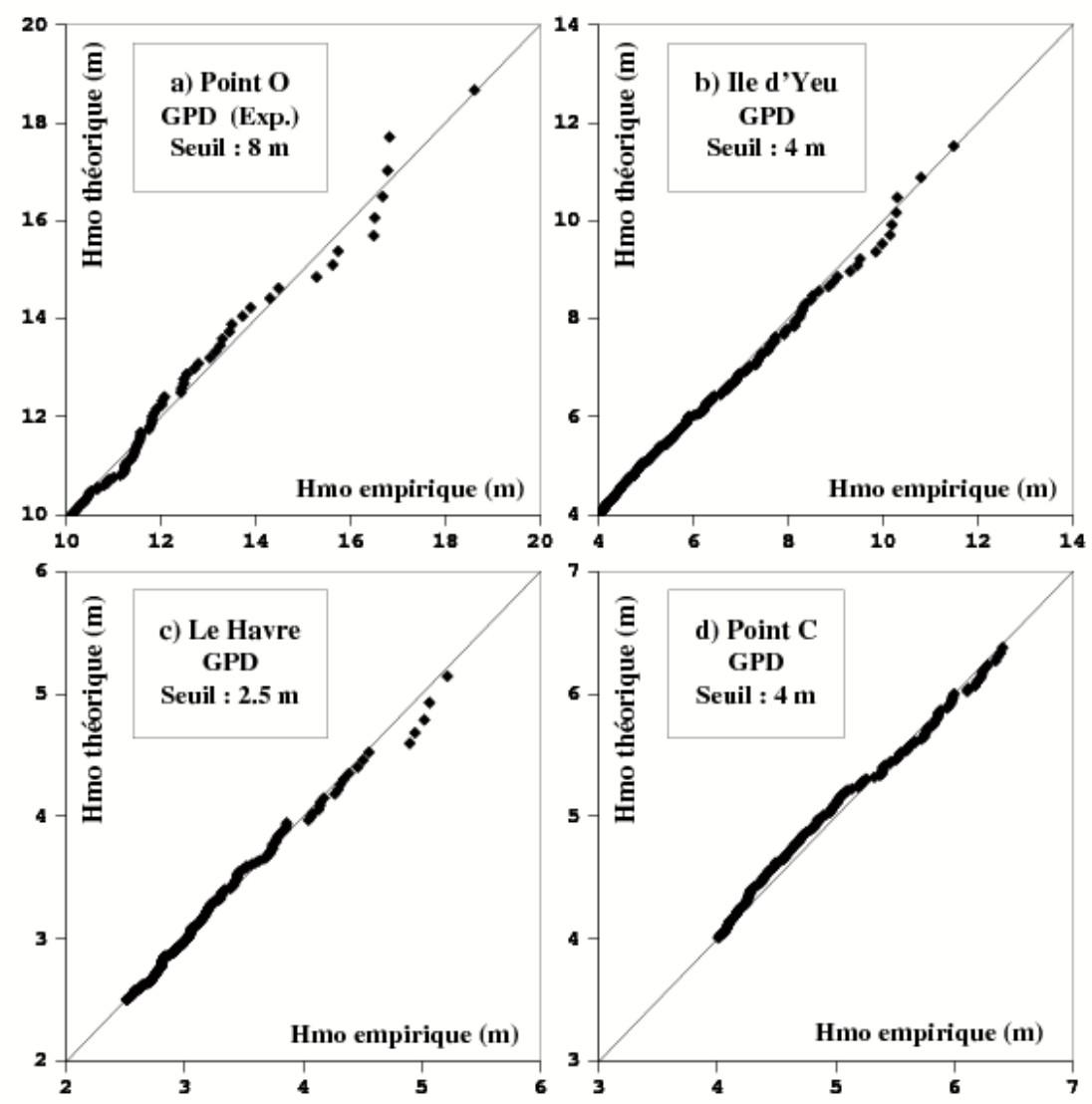

Figure 7: Méthode POT. Diagnostic de l'ajustement à une loi GPD en 4 sites: a) point océanique ; b) Ile d'Yeu ; c) Le Havre ; d) point côtier.

On constate que, dans les deux cas, les ajustements obtenus sont acceptables. De plus, les valeurs estimées du paramètre de forme $\xi$ sont proches, ce qui indique une bonne cohérence entre les deux méthodes. Au point $\mathrm{O}$, les meilleurs ajustements sont obtenus lorsque l'on fixe à 0 la valeur du paramètre de forme, i.e. que l'on considère une loi de Gumbel pour la distribution des maxima annuels et une loi Exponentielle en approche POT. Ces deux distributions ne sont pas bornées. Pour tous les autres points, les valeurs négatives de $\xi$ indiquent que les distributions sont bornées. On constate de plus que la valeur absolue du paramètre de forme augmente (c'est à dire que le caractère borné de la loi est de plus en plus prononcé) à mesure que la profondeur d'eau diminue.

L'approche POT conduit à des ajustements légèrement meilleurs que la méthode des maxima annuels, dès lors que l'on choisit un seuil adapté. Sur chacun des 4 sites, différents seuils ont été testés et comparés sur la base de critères graphiques et de tests d'ajustements statistiques. Le meilleur choix de seuil (correspondant aux seuils indiqués figure 7) se situe entre les quantiles $95 \%$ et $98 \%$ de la série complète de $\mathrm{H}_{\text {mo }}$ pour le point $\mathrm{O}$, l'Ile d'Yeu et Le Havre (en moyenne et grande profondeur) et au niveau du quantile $90 \%$ au point $\mathrm{C}$ (en faible profondeur). L'écart relatif entre les estimations ponctuelles des hauteurs de houle centennales obtenues avec les deux méthodes est de l'ordre de $14 \%$ au point $\mathrm{O}, 8 \%$ à l'Ile d'Yeu, $2 \%$ au Havre et inférieur à $1 \%$ au point $\mathrm{C}$. 
On représente sur la figure 8 les niveaux de retour de la variable $\mathrm{H}_{\mathrm{mo}}$, calculés avec la méthode POT. L'influence de la profondeur d'eau sur la forme de la distribution est à nouveau mise en évidence. En particulier, au point $\mathrm{C}$, les effets du déferlement qui écrête les plus hautes vagues de la distribution sont visibles. Cependant, l'effet de "palier » reproduit par la loi théorique est sans doute trop marqué par rapport à la réalité, dans la mesure où les simulations sont effectuées avec un niveau moyen de mer constant. On atteint donc avec cette gamme de profondeurs les limites du domaine de validité des extrapolations des hauteurs extrêmes, effectuées à partir de cette première version de la base de données d'états de mer (construite avec des conditions hydrodynamiques stationnaires).
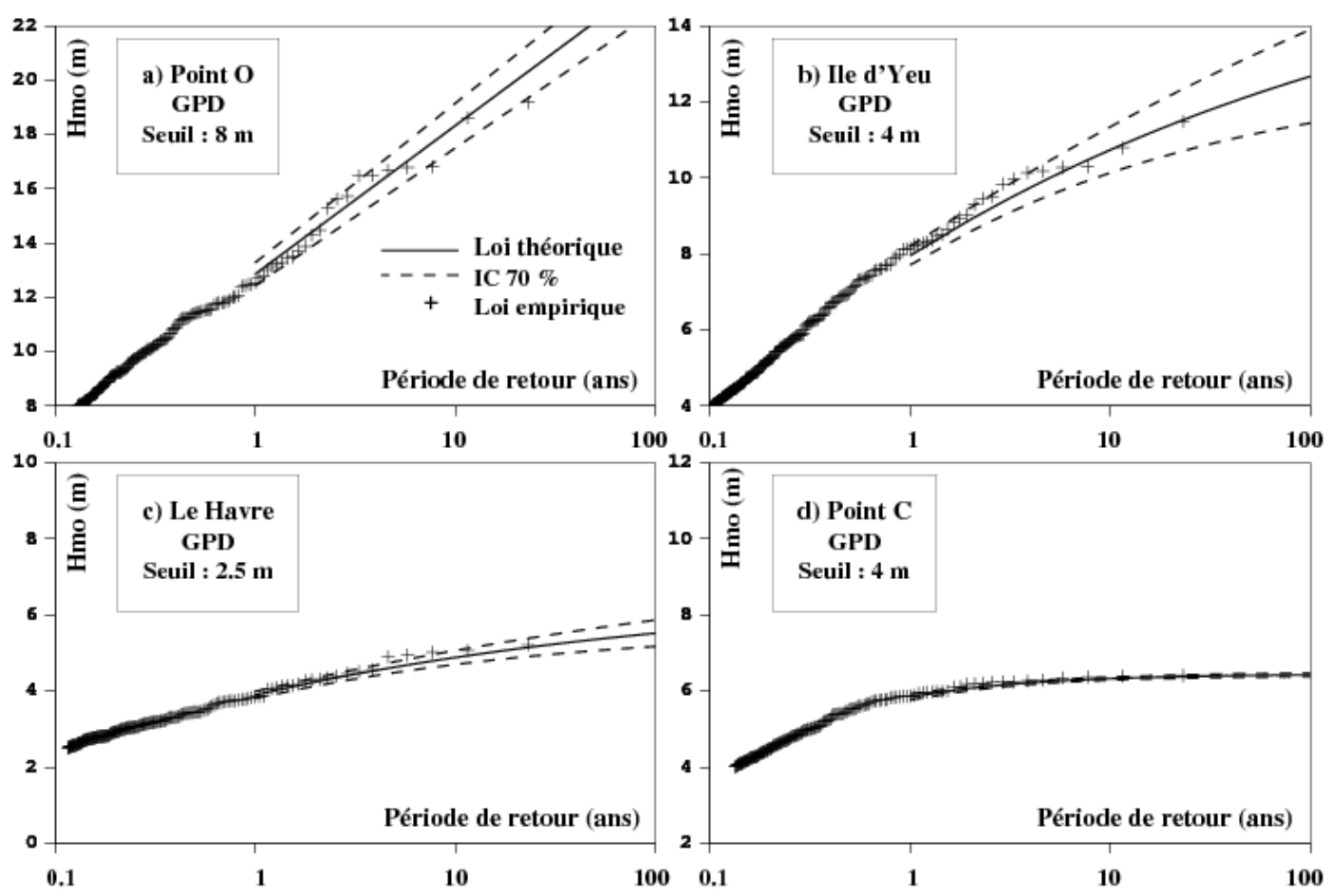

Figure 8: Méthode POT. Niveaux de retour de la hauteur significative spectrale en 4 sites: a) point océanique ; b) Ile d'Yeu ; c) Le Havre ; d) point côtier.

\section{Conclusions et perspectives}

Une base de données d'états de mer a été constituée le long des côtes françaises sur la période 1979-2002, à partir de simulations de hindcast effectuées avec le logiciel TOMAWAC, forcé par les vents issus de la ré-analyse ERA-40 du Centre Européen de Prévision Météorologique à Moyen Terme. Un premier traitement statistique a permis d'établir une cartographie des climats d'états de mer sur le plateau continental européen. Deux méthodes d'estimation statistique des hauteurs extrêmes ont été testées : la méthode dite «des maxima annuels » et une méthode à seuil. Elles conduisent à des résultats comparables sur la sélection de quatre points choisis. Dans les deux cas, l'influence de la profondeur d'eau sur la forme de la distribution des hauteurs extrêmes est nettement mise en évidence. 
L'automatisation de ces deux méthodes d'extrapolation statistique est en cours sur l'ensemble des points des maillages de calcul, de manière à pouvoir établir une cartographie des hauteurs extrêmes de houle le long des côtes françaises. Par ailleurs, l'ouverture d'un site Internet, similaire à $\mathrm{CANDHIS}^{4}$ et dédié à la diffusion des donnée de la base numérique d'états de mer, est prévue d'ici Décembre 2006.

\section{Bibliographie}

1. Benoit M., Marcos F., Becq F., (1996), Development of a third generation shallow water wave model with unstructured spatial meshing. Proc. 25th Int. Conf. on Coastal Eng. (ICCE'1996), Orlando (Floride, USA), 465-478.

2. Benoit M., Lafon F., (2004), A nearshore wave atlas along the coasts of France based on the numerical modelling of wave climate over 25 years. Proc. 29th Int. Conf. on Coastal Eng. (ICCE'2004), Lisbonne (Portugal), 714-726.

3. Bretherton, F.P., Garret C.J.R., (1969), Wavetrains in inhomogeneous moving media. Proc. Roy. Soc. London, Series A, 302, 529-554.

4. CANDHIS, (site web) : www.cetmef.equipement.gouv.fr/donnees/candhis/

5. Coles S., (2001), An introduction to statistical modelling of extreme values. Springer Series in Statistics, Springer Verlag, 208 p.

6. Komen, G.J., Cavaleri L., Donelan M., Hasselmann K., Hasselmann S., Janssen P.A.E.M., (1994), Dynamics and modeling of ocean waves. Cambridge Univ. Press, 532 p. 